\title{
Prevalence of Bacteremia among the HIV and Non HIV Infected Patients Visiting Alupe Sub Hospital, Alupe Division of Teso South Sub-County, Busia County, Kenya between 2017 and 2019
}

\author{
Anne N. Mutsami ${ }^{1}$, Caroline Ngugi ${ }^{1}$, John Kiiru ${ }^{2}$ \\ ${ }^{1}$ Department of Medical Microbiology, School of Biomedical Sciences, Jomo Kenyatta University of Agriculture and Technology, \\ Juja, Kenya \\ ${ }^{2}$ Department of Microbiology, Kenya Medical Research Institute, Nairobi, Kenya \\ Email: annemutsami@gmail.com
}

How to cite this paper: Mutsami, A.N., Ngugi, C. and Kiiru, J. (2021) Prevalence of Bacteremia among the HIV and Non HIV Infected Patients Visiting Alupe Sub Hospital, Alupe Division of Teso South Sub-County, Busia County, Kenya between 2017 and 2019. Open Journal of Medical Microbiology, 11, 249-266.

https://doi.org/10.4236/ojmm.2021.113016

Received: January 31, 2021

Accepted: September 21, 2021

Published: September 24, 2021

Copyright $\odot 2021$ by author(s) and Scientific Research Publishing Inc. This work is licensed under the Creative Commons Attribution International License (CC BY 4.0).

http://creativecommons.org/licenses/by/4.0/

\begin{abstract}
Background: Bacteremia is the presence of viable bacteria in circulating blood. This is common in HIV patients, leading to a significant public health problem worldwide. It is concomitant with upraising cases of antibiotic resistance that results in high morbidity rates leading to increased hospital visits and high mortality rates. Gram-negative bacteria which produce extended-spectrum $\beta$-lactamase (ESBL) are becoming more common and pathogenic in this group of people. Genes responsible for resistance are well-known to spread among various microorganisms. By employing a case-control study design, this study determined prevalence of bacteremia in Salmonella ssp, Klebsiella ssp, and Escherichia coli in HIV positive and HIV negative patients attending Alupe subcounty hospital. Methods: A total of 113 HIV positive and 133 HIV negative patients were sampled purposively grounded on Lameshow sample size determination formula. Consent was sought from the study participants followed by questionnaire administration. Blood $(10 \mathrm{ml})$ was collected from each enrolled patient and later subjected to full haemogram $(1 \mathrm{ml})$, Salmonella Antigen test $(1 \mathrm{ml})$ and general bacterial isolation $(8 \mathrm{ml})$. Results: HIV positive patients experienced significantly different bacterial diseases infection from those who were HIV negative $\left(\chi^{2}=27.684, \mathrm{P}=0.024\right)$. In the current study, total prevalence of Salmonella spp. among HIV positive and HIV negative patients was $11.1 \%$, Klebsiella spp., 18.52\%, Staphylococcus spp., $48.15 \%$, Shigella spp $7.4 \%$ while that of Brucella spp., Neisseria gonorrhea, Aeromonas carviae and Plesomonas shigelloids each was 3.7\%. Escherichia
\end{abstract}


coli was not isolated from any of the blood samples collected from the study participants. Results were made available to policy makers for use in coming up with cognizant measures of prevention of bacteremia, hence a healthy society.

\section{Keywords}

Prevalence, Salmonella ssp, Klebsiella ssp, Escherichia coli Isolates

\section{Introduction}

\section{Background of the Study}

Bacteremia is the presence of viable bacteria in the bloodstream. Bacteremia is a common cause of morbidity and mortality in both high and low income countries, but with variations in the causative organisms and risk factors [1]. Bacteremia is classified as hospital-onset (if it occurs more than $48 \mathrm{~h}$ after admission) or community-onset (if it occurs in less than $48 \mathrm{~h}$ after admission) [2]. Additionally, an epidemiological different group from both community-acquired (CA) and nosocomial infection is the Healthcare-associated infection [3].

Asymptomatic bacteremia may occur during normal daily undertakings such as experiencing minor cuts when carrying out household chores, after minor medical procedures, conducting oral hygiene and others [4]. In healthy individuals, these clinically nonthreatening infections are transitory hence cause no further abnormalcy. This is so because physical barriers such as the skin and non-specific immune defence mechanisms constantly keep bacteria in check [5]. Nonetheless, when the immune system fail or become compromised, bacteremia will develop into bloodstream infection that can progress into many clinical ranges and is differentiated as septicemia [4]. Mucosal lining which is not as strong as the skin may allow a number of primary pathogens to get access, for example some Streptococcus pneumoniae serotypes that can pass via the respiratory mucosal lining, Salmonella typhi via the gastrointestinal lining and Staphylococcus aureus via the mucosa or integument into the blood stream.

Previous studies have indicated that documented information on prevalence and causes of community-acquired bloodstream infections in Africa are rare. Twenty two legible studies on bacteremia where 58, 296 patients were described (43,130 children and 15,166 adults). From these previous studies, a total of 2051 (13.5\%) of 15, 166 adults and 3527 (8.2\%) of 43,130 children had bloodstream infections. Some of the infections were due to malaria, those who had non-malarial infection $(1643 ; 29.1 \%)$ has infections cause predominantly by Salmonella enterica while $(1031 ; 18.3 \%)$ of the infections were as a result of $\mathcal{S}$. pneumoniae, which was most commonly isolated in children. Other isolates commonly found were S. aureus (531 infections; 9.5\%) and Escherichia coli (412; 7.3\%) [6]. Klebsiella and Actinobacter are also other important gram-negative bacteria clinically, with Klebsiella pneumoniae, bloodstream infection having a mortality rate of 
about $20 \%$ [3].

Bacteremia has been reported to be common in HIV-infected patients due to immunosuppression. Although there is a reduction in AIDS-related deaths and opportunistic infection rate as a result of the introduction of combined antiretroviral therapy (cART), infection with the human immunodeficiency virus (HIV) remains a cause of increased risk of bloodstream infection (BSI) [7]. In vitro experiments showed that infection with HIV leads to productive infection of cluster of differentiation $4^{+}\left(\mathrm{CD} 4^{+}\right) \mathrm{T}$ cell that culminate to either cell lysis or giant cell/syncytia formation, where both infected and uninfected cells fuse, hence spread of infection. The same was reported to happen in humans infected with HIV where depletion of GALT $\mathrm{CD}^{+}$reservoirs was reported. Depletion of CD4+ T cells leads to immunosuppression hence onset of opportunistic infections especially bacteremia. There occurs immunesuppression hence opportunistic infections set in more so bacteremia [8]. High mortality rates reported in HIV infected patients have been associated with blood stream infections [9].

All bacterial infections are treated using antibiotics, unfortunately there have been reported clinical failures attributed to antibiotic resistance [10]. Increase in the population of antibiotic resistant bacteria poses a risk to the sustained use of antibiotics to treat bacterial infections [11].

Globally, antibiotic resistance portends the effective prevention and treatment of an ever-increasing range of infections caused by bacteria [12]. Widespread use of cotrimoxazole prophylaxis against opportunistic infections in HIV/AIDS patients has been attributed to bacterial resistance [13]. Cotrimoxazole prophylaxis has been used for several years but little is known about its impact on the spread of antibiotic resistance in HIV positive patients [13]. There are several gaps which still exist on how to enforce policies on how to contain antibiotic resistance. A prevalence of $22.9 \%$ of blood stream infection was recorded in HIV infected patients on highly active antiretroviral therapy [14]. Gram negative bacteria have been predominant pathogens isolated from HIV positive and negative patients (Mootsikapun, 2007b). Research indicates that, prevalence of bacteremia vary depending on season, region and is mainly associated with differences in population demographic and risk factors [15]. However, there is no documented information on prevalence of bacteremia in Busia County.

Alupe hospital research background owing to its close proximity to KEMRI/ KALRO attracts more patients in view for further investigations and also acts as a referral centre due to its quality treatment. As at the time of study commencement, the Comprehensive Care Centre had an enrolment of 1382 patients (Alupe hospital CCC record as per December 2017). This made the hospital a more suitable area for the study since it was convenient for analyzing samples at the KEMRI laboratories and was time saving. However, bacteremia is one of the commonest causes of death in patients attending Alupe Sub County hospital especially in HIV cohorts, since the socioeconomic status in- 
dicated the population is predisposed to most infections and this has alluded to the high poverty index, poor sanitation and unimproved water sources. The process of seeking for answers to the specific study questions culminated to a clear comparison of certain parameters in the two cohorts. Among these parameters was prevalence of bacteremia. As such, lack of documented information on the prevalence of bacteremia in HIV positive patients in Alupe and in Busia County as a whole prompted the need to carry out the study. This has lead to robust investigations of bacteremia, its etiologic factors and assessment of antimicrobial susceptibility profiles of isolated bacterial pathogens. This report will aid in updating the choice of empirical treatment. Results of this study have provided critical data to care givers and health planners in preventing the occurrence and spread of bacteremia and resistance strains hence boosting the life expectancy of people living with HIV. The research was built on a research question of "what is the prevalence of bacteremia in HIV and non HIV infected patients visiting Alupe hospital?" Hence the objective was to determine the prevalence of bacteremia among the HIV and non HIV infected patients visiting Alupe hospital.

\section{Methods}

\subsection{Study Site}

The study was carried out in Alupe Sub county hospital, Alupe division of Teso South sub-county, Busia County, Kenya. It is located approximately $6 \mathrm{~km}$ from Busia town along Busia-Malaba road. The Hospital is $50 \mathrm{~m}$ away from Kenya Medical Research Institute/ Centre for Infectious and Parasitic Diseases Control Research (KEMRI/CIPDCR) with a Comprehensive Care Centre (CCC) that enrolls HIV patients across the county making it suitable site for the study Alupe division serves as the main catchment area of Alupe Sub-District Hospital within Kenya. However, other neighbouring divisions such as Amukura division in Teso North Sub County, Busia Sub County in Kenya and the neighbouring Busia district in Uganda are served by the facility. This is a level 4 facility with a catchment population of 40,000 peoples offering both in-patient and out-patient services. The population served is multi-ethnic although Teso is the predominant ethnic group. Busia County experiences a tropical climate which is influenced by the presence of Lake Victoria. Annual temperature ranges from $17^{\circ} \mathrm{C}$ to $30^{\circ} \mathrm{C}$ with an average range of $24^{\circ} \mathrm{C}$ to $26^{\circ} \mathrm{C}$. Mean annual rainfall ranges between $900-1500 \mathrm{~mm}$ distributed throughout the year. Long rains are experienced in the month of March until June whereas short rains span the month of September and October.

\subsection{Study Population}

The study population included HIV positive patients enrolled at Alupe Sub County Hospital CCC and HIV negative patients who visited Alupe Sub county hospital for other health related problems other than HIV management. The catchment areas included; the whole of Busia County, other neighboring coun- 
ties and the neighboring Country Uganda. At the beginning of this study, the facility had enrolled 1382 patients in the Comprehensive Care Centre (CCC).

\subsection{Study Design}

The study employed a case control research design. Cases were HIV positive patients who presented with symptoms of bacteremia. Symptoms of bacteremia varied ranging from fever, rapid heart rate, shaking chills, low blood pressure, gastrointestinal symptoms (such as abdominal pain, nausea, vomiting, and diarrhea), rapid breathing, and/or change in mental status such as confusion. Controls were patients who tested negative of HIV at recruitment point but present with symptoms of bacteremia. Therefore, this study design compared patients who had disease or outcome of interest, that was HIV (cases) with patients who did not have the disease or outcome that was non HIV (controls).

\subsection{Inclusion Criterion}

\subsubsection{Inclusion Criteria for HIV Positive Patients}

Included in the study were:

1) Patients who had enrolled at the CCC for care and presented with symptoms of Salmonella, Klebsiella and Escherichia coli infection. Such general symptoms included; Abdominal cramps, abdominal pain, fever, muscle ache, neck stiffness, sensitivity to bright lights, chills, rash, light-headedness, altered mental states, flu-like symptoms, cough, which may produce mucus that is yellow, green, or bloody, breathing difficulties sudden severe watery diarrhea that may change to bloody stools, gas, loss of appetite or nausea, fatigue and at times vomiting.

2) Those patients who were 12 years old and above and consented to participate in the study.

3) Patients who consented to have their blood stored for more than 30 days and investigation carried out on it.

3) Patient $s$ who visited the facility for treatment but upon testing, turned positive for the virus.

\subsubsection{Inclusion Criterion for HIV Negative Patients}

1) Patients who had visited Alupe Sub county hospital for medication purposes and were 12 years and above.

2) Patients who were confirmed to be HIV negative but presented with symptoms of Salmonella, Klebsiella and Escherichia coli infections such as abdominal cramps and abdominal pain fever, muscle ache, neck stiffness, sensitivity to bright lights, chills, rash, light-headedness, and altered mental states, flu-like symptoms, cough, which may produce mucus that is yellow, green, or bloody, breathing difficulties sudden severe watery diarrhea that may change to bloody stool, gas, loss of appetite or nausea, fatigue and sometimes vomiting.

3) Patients who gave signed informed consent to participate in the study.

4) Patients who consented to have their blood stored for more than 30 days and investigation carried out on it. 


\subsection{Exclusion Criterion}

\subsubsection{Exclusion Criteria for HIV Positive Patients?}

Excluded from the study were:

1) Patients who had visited the CCC for routine care such as to collect antiretroviral drugs, pregnancy tests and/or counselling.

2) Patients who were critically anaemic such that $10 \mathrm{ml}$ of blood could not be drawn from their blood system.

3) Patients who were in critical health condition.

4) Patients who were mentally ill.

\subsubsection{Exclusion Criterion for HIV Negative Patients Same Applies Here}

1) Patients who visited the hospital for personal reasons or non-health related issues or private arrangements.

2) Patients who did not present with symptoms of Salmonella, Klebsiella and Escherichia coli infections.

3) Patients who tested positive for HIV test.

4) Patients who were critically anaemic such that $10 \mathrm{ml}$ of blood can not be drawn from their blood system.

5) Patients who were in critical health condition.

6) Patients who were mentally ill.

\subsection{Recruitment Procedure}

Patients were received by the clerking officer who then directed the patient to the clinician. The clinician engaged the patient in some enquiry related to the health condition presented. Once the Clinician confirmed that the patient met the criteria, the patient was then asked whether he/she consented to take part in a study whose objective was explained to the patient. Upon giving consent, the clinician took the patient through the questionnaire. The clinician referred the patient to the laboratory for tests. Among the samples taken were $10 \mathrm{mls}$ of blood for blood culture, salmonella antigen test and full haemogram test.

Upon return from the laboratory with the results, the clinician prescribed the drugs basing on the results. The clinician directed the patient to the pharmacy/and or to the injection room as it deemed necessary. The patient was then released to go home.

\subsection{Sample Size}

Sample size was determined using [16] formula. The total samples used were from 246 patients.

\subsection{Sampling Method}

Cases were purposively sampled from the HIV positive patients who were registered at the CCC for HIV management. As these patients reported to the CCC for care, those patient who were 12 years and above presented with symptoms of Salmonella, Escherichia coli and Klebsiella infections were talked to about the 
study. Symptoms included; poor appetite, abdominal pain, headache, generalized body aches and pain, high fever and or with chills, diarrhea or constipation and fatique. The clinician explained to the patient the nature and purpose of the study that was to be undertaken and asked the patient if he/she is was willing to participate in the study. Those who consented, were given a consent form to sign witnessed by the clinician. The patient who consented was given a questionnaire to fill. The patient proceeded to the laboratory for blood and other sample collection as indicated by the clinician.

Control cases were purposively sampled from patients who visited the hospital for treatment of symptoms related to Salmonella, Klebsiella and Escherichia coli infections. A patient who was 12 year and above presenting with symptoms of Salmonella, Escherichia coli and Klebsiella infections, the clinician explained to $\mathrm{him} / \mathrm{her}$ about the study, its nature and purpose. Symptoms included; poor appetite, abdominal pain, headache, generalized body aches and pain, high fever and or with chills, diarrhea or constipation and fatique. The clinician counseled the patient on the importance of knowing his/her HIV status. A patient who consented testing for HIV and taking part in the study was given a consent form to sign as the clinician witnessed. The patients were referred to the VCT for further counseling and testing for HIV status. The results were taken back to the clinician. Patient who turned out to be HIV positive were referred to the CCC for care. The patient was given a questionnaire to fill after which he/she proceeded to the laboratory for blood and other sample collection depending on the clinician's request. Each questionnaire had a unique number (code) as designed by the study team for privacy. Patient's study unique number (patient ID), patient's location (room and bed number in case of in patients), date and time of collection, collector's initials and site of collection was filled in the collection chart form.

\subsection{Data Collection}

\subsubsection{Questionnaire}

A structured questionnaire was administered by the PI to gather the socio-demographic data, clinical and lifestyle factors of the study participants such as age, education level, occupation, marital status, history of bacteremia and recent history of treatment and hospitalization among others. Names were not indicated on the form but a unique bar code used. A separate file with names and contacts of the participants for follow up after the results was maintained by the principle investigator.

\subsubsection{Sample Collection and Processing}

Blood samples were collected, incubated in blood culture for growth, subcultured on solid media, followed by gram staining, then finally biochemical tests were carried out for identification.

A volume of $10 \mathrm{ml}$ venous blood was collected from participants using a needle (gauge 21$)$ and a syringe ( $10 \mathrm{mls}$ size). This was done by a trained phlebotomist. Wearing gloves, the phlebotomist wraped a band at the top of the left 
arm, sterilized the site of blood collection using a sterile cotton swab soaked in methylated spirit then drew blood from the vein. After blood collection, a sterile spirit pad was used to sterilize the venipuncture site as the needle was removed. A total of $8.0 \mathrm{mls}$ of blood was added to blood culture medium. This culture medium containing blood sample was incubated immediately at $37^{\circ} \mathrm{C}$ for $7-14$ days. Of the remainder, $1.0 \mathrm{ml}$ was placed in a lavender top vacutainer for full haemogram which was done within 30 minutes of blood collection. The remaining $1.0 \mathrm{ml}$ was released into a red top microvacurtainer for salmonella antigen test. Needles were disposed off in an appropriate container that had been well labeled "sharp objects". While other used materials like gloves and paper towels were disposed off in a separate labeled container.

The patient was advised on how to take care of the venipuncture site to avoid any complications arising. This included: to temporarily carry items with the opposite arm, to avoid lifting or carrying heavy objects for about 8 hours, avoid activities that would put stress on the puncture site like digging within 8 hours after the procedure.

\subsubsection{Full Haemogram Procedure}

Immediately after blood collection, $1.0 \mathrm{ml}$ of blood was placed in a lavender top vacutainer. This was run in a Backman Coulter MAXM machine (Beckman Coulter manual 2011) for complete blood count (CBC) as per the CDC laboratory manual. Parameters of interest were white blood cell count, absolute neutrophil count, absolute band count, monocytes and procalciton. These parameters were compared with the normal range to diagonise a possible bacterial infection. From the results of full haemogram, the clinician took up the management of the patient.

\subsubsection{Salmonella Antigen Test}

The $1.0 \mathrm{ml}$ of blood was subjected to salmonella antigen test. Using qualitative slide agglutination test to determine presence of Salmonella antigens. The screening test was done to ascertain the presence of Salmonella spp. in the blood sample. All reagents were brought to room temperature and well mixed. To each reaction well labeled "O" and " $\mathrm{H}$ ", one drop $(25 \mu \mathrm{l})$ of the serum was added. To the reaction Well labeled positive control (PC) and negative control (NC), $25 \mu \mathrm{l}$ of the PC and $25 \mu \mathrm{l}$ of the NC was added respectively. Antigen solutions of salmonella typhi "O" and Salmonella typhi " $\mathrm{H}$ " was added to the Well labeled "O" and " $\mathrm{H}$ " respectively. This was mixed thoroughly using an applicator stick and gentle rotation of the slide. The slides were observed for agglutination. Positive test was the slides that showed agglutination in 1 minute. Negative results were the slides that did not show agglutination within 1 minute [23].

\subsubsection{Isolation of Salmonella spp, Klebsiella spp and Escherichia coli from Blood Samples}

Blood that had been inoculated into the blood culture medium were incubated at $37^{\circ} \mathrm{C}$ for 7 - 14 day. The medium was checked for growth (turbidity) starting 
from the third day after incubation. Any culture that showed turbidity was sub cultured on MacConkey agar (Oxoid). A sterile wire loop was used to transfer an aliquot of the turbid culture and streaked on Macconkey agar (Oxoid). This was incubated at $37^{\circ} \mathrm{C}$ for $12-24 \mathrm{hr}$. after which the agar was inspected for any growth. Basing on the colony morphological appearance and colour, any suspected colony for Salmonella spp, Klebsiella ssp, and Escherichia coli was sub cultured on MacConkey separately. These were incubated at $37^{\circ} \mathrm{C}$ for $12-18 \mathrm{hr}$ to yield pure colonies of Salmonella spp, Klebsiella spp, and Escherichia coli. Any suspected colony for Salmonella spp (colorless colonies with no precipitated zone) was sub cultured on Salmonella Shigella agar (SS) for further purity and incubated at $37^{\circ} \mathrm{C}$ for $12-24 \mathrm{hr}$ to yield pure colonies of Salmonella.

\subsubsection{Gram Staining for Identification}

Basing on the colony morphology and colour in reference to the manual [17], pure colonies of suspected Salmonella spp, Klebsiella spp and Escherichia coli were subjected to Gram staining technique [17] to determine whether the organism is gram positive or gram negative.

Using a sterile wire loop, a small amount of a colony was collected from the agar. A thin smear was made on a sterile microscope slide. The smear was air dried and heat fixed by passing the slide containing the smear over a lit burnsen burner. The smear was flooded with crysral violet stain for 30 - 60 seconds, then followed by washing using distilled water in a wash bottle. The smear was then flooded with Lugol's iodine for 30 - 60 seconds, and further washed using distilled water in a wash bottle. Water was tiped off and the smear was decolourised rapidly (a few seconds) using acetone and washed immediately using distilled water in a wash bottle. The smear was flooded with neutral red stain for 2 minutes. The smear was washed with distilled water and the back of the slide was wiped using a piece of clean tissue and placed in a draining rack for the smear to dry. After drying, the smear was examined under X100 oil immersion objective lens. Those cells that appeared blue or purple were recorded as gram positive while those that appeared red or pink were reported as gram negative. Apart from colour of cells, the morphology of bacteria was reported either as rods or cocci, single cells, double cells, in chain or in clusters.

\subsubsection{Biochemical Tests}

Biochemical tests were carried out for further identification and confirmation of Salmonella spp, Klebsiella spp and Escherichia coli. Biochemical characteristics identification was done using five tube method media (Oxoid Ltd., Basingstoke, United Kingdom) of Triple sugar iron agar, Sulphur indole motility agar, Simmon's citrate agar, MRVP broth and urea agar and their reaction to oxidase reagent ( $1 \%$ dimethyl-phenylene-diamine dihydrochloride).

All the biochemical tests were performed as per the manual [17]. From the previous procedure of sub culture which yielded pure colonies, using a sterile wire loop/stab wire, a small amount of a bacterial colony was collected. The inoculum 
was introduced on/into the various biochemical media. Besides the test organism, the biochemical media was inoculated separately with Escherichia coli ATCC 25922 as a control experiment. These biochemical procedures were as described below.

\section{1) Triple Sugar Iron (TSI) Test}

Using a sterile straight inoculation needle, the top of a well isolated colony was touched. The TSI slant was first inoculated by stabbing through the centre of the medium up to the bottom of the tube then streaking the surface of the agar slant as the needle was drawn out of the media. The cap of the tube was screwed loosely. The tube was incubated at $37^{\circ} \mathrm{C}$ for $18-24 \mathrm{hr}$. Tubes were inspected to confirm which carbon source had been utilized. The abbreviations that were used were as follows: $\mathrm{A}=$ Acid; $\mathrm{K}=$ Alkaline; $\mathrm{H}_{2} \mathrm{~S}=$ Hydrogen sulfide produced; $\mathrm{N}=$ No change; Plus (+) $\mathrm{H}_{2} \mathrm{~S}$ meaning production of $\mathrm{H}_{2} \mathrm{~S}$, minus $(-) \mathrm{H}_{2} \mathrm{~S}$ meaning no $\mathrm{H}_{2} \mathrm{~S}$ production. Red/Red = Red slant and red butt, Red/Yellow $=$ Red slant and yellow butt, Yellow/Yellow = yellow slant and yellow butt.

\section{2) Catalase Test}

A drop of hydrogen peroxide was places on a sterile microscope slide. Using a sterile wire lope a small amount of a colony was collected from a colony of 18 $24 \mathrm{hr}$ old pure bacteria culture. The colony amount was place in the hydrogen peroxide. Results were considered to be positive if there was immediate bubbling and liberation of oxygen. Negative results were recorded if there was no immediate bubbling and no liberation of oxygen.

\section{3) Urease Test}

This test was done to determine whether the bacteria were able to hydrolyse urea to produce ammonia and carbon (IV) oxide by use of urease enzyme. Urease broth was inoculated with a heavy inoculum from a $12-24 \mathrm{hr}$ old pure culture by streaking the entire slant. Tubes were incubated at $37^{\circ} \mathrm{C}$ for 6 days. Observations were made after $6 \mathrm{hr}, 24 \mathrm{hr}$ and then daily upto the 6th day. Urease production was indicated by a bright pink colour on the slant. Any degree of pink was considered as a positive result. This colour may extend slightly into the butt.

\section{4) Sulphur Indole Test}

This was done to test for the microbe's ability to split the amino acid tryptophan and form compound indole. By use of a sterile wire loop, a colony from the previous culture was inoculated into the broth. This was incubated at $37^{\circ} \mathrm{C}$ for $24-48 \mathrm{hr}$. An aliquot $(0.5 \mathrm{ml})$ of Kovac's reagent was added to the broth culture. OBacteria possessing tryptophanase enzyme, were able to convert tryptophan amino acid in the medium to indole, pyruvic acid, ammonium (NH4+) and energy. Indole reacts with Kovac's Reagent (contains hydrochloric acid and p-dimethylaminobenzaldehyde in amyl alcohol) forming a red rosindole dye. Since amyl alcohol is not water soluble, the red dye forms in an oily layer, observed as a ring at the top of the medium. Such observations were interpreted as indole $(+)$ whereas the medium that did not form a red ring on the surface was interpreted as indole (-). Organisms once identified were stocked in $1 \mathrm{ml}$ capacity vials containing Mueller Hinton broth (Oxoid) containing 15\% (v/v) glycerol 
and stored at $-70^{\circ} \mathrm{C}$ for further analysis [18].

\section{Results}

\subsection{Data Analysis}

Data analysis was conducted using SPSS version 22 and STATA version 16. Categorical data were tabulated. Mean median and standard deviation were used to summarize the data. Comparisons of means and medians were done using Student's t test and Mann Whitney U test respectively. Prevalence rates were determined using descriptive statistics (e.g. proportions). Tests of associations were performed using Chi-square test for categorical variables. Student's t-test was performed for continuous variables, and Fisher's exact test was performed for nominal variables. A P-value $\leq 0.05$ was considered significant. Descriptive statistics were used to describe clinical and socio demographic characteristics. Data was presented using pie charts, bar graphs, line graphs and tables.

\subsection{Ethical Statement}

For quality data, participants were explained to clearly what the study was all about and how to fill the questionnaire. Standard operating Procedures were adhered to while carrying out laboratory procedures. Concent was sort from participants and any information given by a participant was kept confidentially. Participants were enrolled in the study willingly and they were not exposed to any harm. This study report is of its own kind in Alupe Busia County.

\subsection{Prevalence of Bacteremia among the HIV and Non HIV Infected Patients Visiting Alupe Hospital}

A total of 246 patients among those who visited Alupe Hospital participated in this study.

\subsubsection{Trend of Drug Regimen among the Population}

Out of this number, 113 (45.9\%) were HIV positive while 133 (54.1\%) were HIV negative patients. For the HIV positive patients, most of them were currently on the first drug regimen (80.5\%). The rest were on the second (11.5\%) regimen while $1.8 \%$ were newly diagnosed and the results showed that $1.8 \%$ of the participants were not yet on any treatment (Table 1).

Table 1. Drug regimen of the HIV positive patients.

\begin{tabular}{cccccc}
\hline & \multicolumn{5}{c}{ Current HIV drug regime } \\
\cline { 2 - 6 } & Newly diagnosed & First & Second & Not on treatment & No response \\
\hline HIV Positive & $2(1.8 \%)$ & $91(80.5 \%)$ & $13(11.5 \%)$ & $2(1.8 \%)$ & $5(4.4 \%)$ \\
\hline
\end{tabular}

\subsubsection{Trend of Drug Regimen among the Population}

The trend of population drug regimen among the population adopted a sigmoid shape. More of the patients were HIV patients on the first regimen. Relatively 
fewer patients were newly diagnosed and were not on drug yet whereas the number reduced in the second regimen. This fitted a polynomial trend having an $\mathrm{R}^{2}$ value of $100 \%$ with an equation depicted by (Figure 1 ):

$$
y=-37.733 x^{3}+265.75 x^{2}-533.12 x+306.9 .
$$

\subsubsection{Prevalence of Bacteremia}

A total of 27 bacteria were isolated from a total sample size of 246, translating to a bacteremia prevalence of $11 \%$. Escherichia coli which was one of the three bacteria of interest was not isolated in any of the samples. A total of eight (8) different types of bacteria were isolated (Table 2) with a difference in prevalence of each type of bacteria species. Individual bacteria prevalence out of the 27 bacteria was as shown below (Table 2).

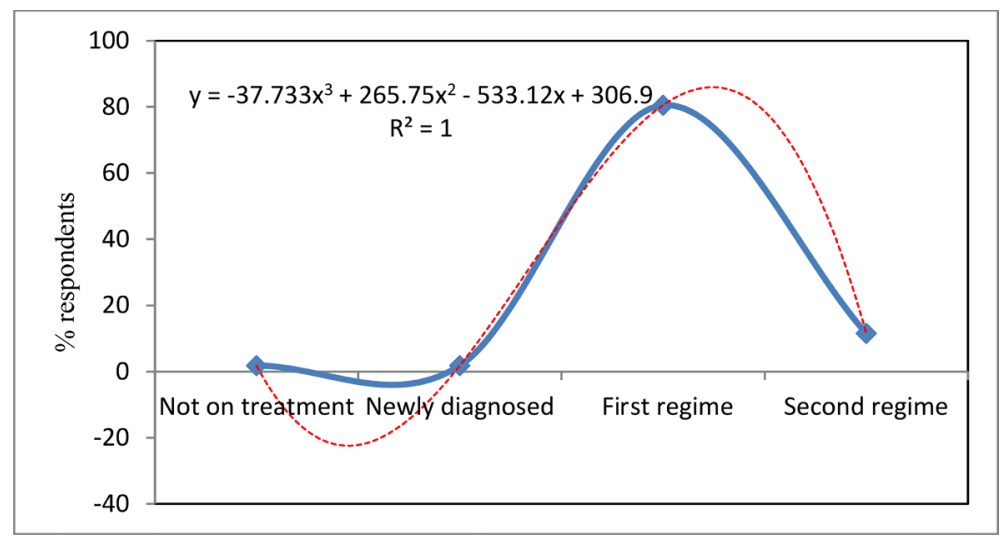

Figure 1. Trend of drug regimen.

Table 2. Prevalence of bacteremia in HIV and Non HIV Positive patients.

\begin{tabular}{clcccc}
\hline S/No & \multicolumn{1}{c}{ Bacteria Species } & $\begin{array}{c}\text { Number of } \\
\text { bacteria }\end{array}$ & (P) & $\begin{array}{c}\text { \% P in HIV } \\
\text { positive }\end{array}$ & $\begin{array}{c}\text { \% P in HIV } \\
\text { negative }\end{array}$ \\
\hline 1 & Staphylococcus spp & 13 & 48.1 & 44.4 & 3.7 \\
2 & Klebsiella spp & 5 & 18.5 & 7.4 & 11.1 \\
3 & Salmonella spp & 3 & 11.1 & 3.7 & 7.4 \\
4 & Shigella spp & 2 & 7.4 & 3.7 & 3.7 \\
5 & Brucella spp & 1 & 3.7 & 3.7 & 0 \\
6 & Neisseria gonorrhea & 1 & 3.7 & 3.7 & 0 \\
7 & Aeromonas caviae & 1 & 3.7 & 0 & 3.7 \\
8 & Plesomonas shigelloids & 1 & 3.7 & 0 & 3.7 \\
& $\quad$ Total & 27 & 100 & 66.7 & 33.3 \\
\hline
\end{tabular}

Prevalence of bacteremia in HIV positive patience was higher $(66.7 \%)$ as compared with that in HIV in HIV negative patients (33.3\%). Using paired sample $\mathrm{t}$-test revealed that there was statistical difference of bacteremia in HIV positive and non HIV positive patients $(t=2.646 \mathrm{p}=0.0331)$. These results were in line with results of the same study where bacterial diseases infecting HIV and 
non HIV patients, diseases experienced by HIV positive were significantly different from those experienced by HIV negative patients $\left(\chi^{2}=27.684, \mathrm{P}=0.024\right)$

\subsubsection{Bacterial Diseases infected by Inpatients.}

Respondents who had been recently taken as inpatients had mainly suffered from Typhoid in this Hospital (55.6\%). A number of the patients $(22.2 \%)$ had Pneumonia and fewer of them had Typhoid + Menengitis while others had Typhoid + pneumonia infection (Figure 2).

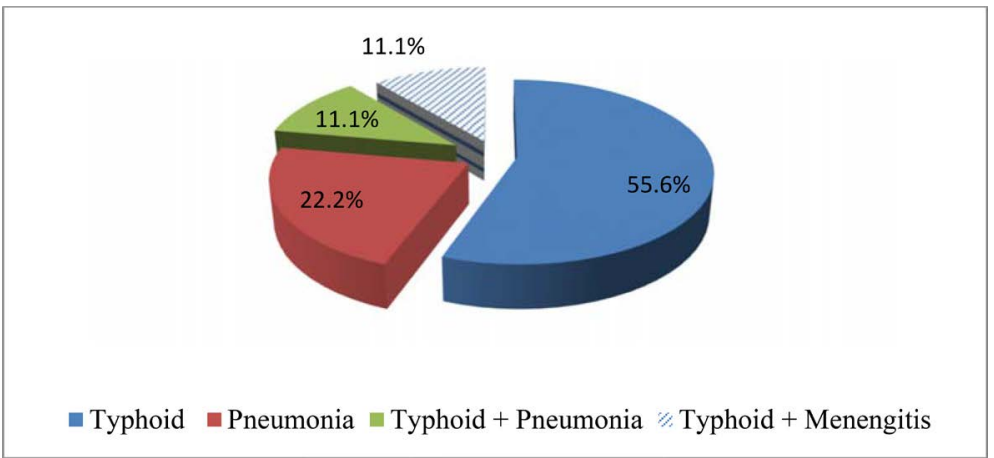

Figure 2. Recent bacterial diseases experienced by inpatients.

\subsubsection{Bacterial Diseases Experienced by HIV positive and HIV negative} Patients.

Typhoid infection was the highly recorded infection in both HIV positive (27.4\%) and HIV negative patients (30.8\%). More HIV positive patients had pneumonia infection (13.3\%) as compared to the same infections in HIV negative patients (3.8\%). The diseases experienced by the HIV positive were significantly different from those experienced by HIV negative patients $\left(\chi^{2}=27.684, \mathrm{P}\right.$ $=0.024)($ Table 3$)$.

Table 3. Diseases experienced by both HIV positive and HIV negative patients.

\begin{tabular}{lccc}
\hline \multirow{2}{*}{ Bacterial diseases } & \multicolumn{2}{c}{ HIV Status } & \multirow{2}{*}{ Total } \\
\cline { 2 - 3 } & Positive (n/\%) & Negative (n/\%) & \\
\hline Pyelonephritis & $1(0.9 \%)$ & $1(0.8 \%)$ & $2(0.8 \%)$ \\
Typhoid + Meningitis & $2(1.8 \%)$ & $0(0.0 \%)$ & $2(0.8 \%)$ \\
Typhoid & $31(27.4 \%)$ & $41(30.8 \%)$ & $72(29.3 \%)$ \\
Pneumonia & $15(13.3 \%)$ & $5(3.8 \%)$ & $20(8.1 \%)$ \\
Brucella & $0(0.0 \%)$ & $2(1.5 \%)$ & $2(0.8 \%)$ \\
Not Indicated & $9(8.0 \%)$ & $11(8.3 \%)$ & $20(8.1 \%)$ \\
Typhoid + Pneumonia & $5(4.4 \%)$ & $5(3.8 \%)$ & $10(4.1 \%)$ \\
Arthritis & $0(0.0 \%)$ & $1(0.8 \%)$ & $1(0.4 \%)$ \\
Pyelonephritis + Typhoid + Pneumonia & $1(0.9 \%)$ & $0(0.0 \%)$ & $1(0.4 \%)$ \\
Total & $113(100 \%)$ & $133(100 \%)$ & $246(100 \%)$ \\
Chi-square value & 27.685 & & \\
P value & 0.024 & & \\
\hline
\end{tabular}




\subsection{Demographic Information of the Sampled Participants}

Most of the respondents were females (69.1\%) and majorities (27.6\%) were in the ages of $41-50$ years. Concerning their marital status, the study found out that $61.8 \%$ were married, $19.1 \%$ were separated/widowed while $16.7 \%$ were single. One participant was currently cohabiting. Out of 246 patients, $6.9 \%$ had no formal education, $8.1 \%$ had tertiary education, $20.7 \%$ had secondary, while majority, $61.4 \%$ had primary as their highest levels of education (Table 4).

Table 4. Sampled Non HIV and HIV infected patient's demographic data.

\begin{tabular}{|c|c|c|c|}
\hline Demographic information & $\begin{array}{l}\text { HIV positive } \\
\quad(\mathrm{n}=113)\end{array}$ & $\begin{array}{l}\text { HIV negative } \\
\quad(\mathrm{n}=133)\end{array}$ & $\begin{array}{c}\text { Total } \\
(n=246)\end{array}$ \\
\hline \multicolumn{4}{|l|}{ Gender } \\
\hline Male & $37(32.7 \%)$ & $37(27.8 \%)$ & $74(30.1 \%)$ \\
\hline Female & $75(56.4 \%)$ & $95(71.4 \%)$ & $170(69.1 \%)$ \\
\hline None response & $1(0.9 \%)$ & $1(0.8 \%)$ & $2(0.8 \%)$ \\
\hline \multicolumn{4}{|l|}{ Age (years) } \\
\hline $11-20$ & $5(4.4 \%)$ & $16(12.0 \%)$ & $21(8.5 \%)$ \\
\hline $21-30$ & $7(6.2 \%)$ & $29(21.8 \%)$ & $36(14.6 \%)$ \\
\hline $31-40$ & $31(27.4 \%)$ & $15(11.3 \%)$ & $46(18.7 \%)$ \\
\hline $41-50$ & $47(41.6 \%)$ & $21(15.8 \%)$ & $68(27.6 \%)$ \\
\hline $51-60$ & $17(15.0 \%)$ & $25(18.8 \%)$ & $42(17.1 \%)$ \\
\hline Above 60 & $6(5.3 \%)$ & $25(18.8 \%)$ & $31(12.6 \%)$ \\
\hline None committal & $0(0.0 \%)$ & $2(1.5 \%)$ & $2(0.8 \%)$ \\
\hline \multicolumn{4}{|l|}{ Current status } \\
\hline Married & $69(61.1 \%)$ & $83(62.4 \%)$ & $152(61.8 \%)$ \\
\hline Single & $11(9.7 \%)$ & $30(22.6 \%)$ & $41(16.7 \%)$ \\
\hline Separated/widowed & $28(24.8 \%)$ & $19(14.3 \%)$ & $47(19.1 \%)$ \\
\hline Cohabiting & $1(0.9 \%)$ & $0(0.0 \%)$ & $1(0.4 \%)$ \\
\hline Child & $1(0.9 \%)$ & $0(0.0 \%)$ & $1(0.4 \%)$ \\
\hline None respondents & $3(2.7 \%)$ & $1(0.8 \%)$ & $4(1.6 \%)$ \\
\hline \multicolumn{4}{|l|}{ Highest level of education } \\
\hline No formal education & $3(2.7 \%)$ & $14(10.5 \%)$ & $17(6.9 \%)$ \\
\hline Primary & $87(77.0 \%)$ & $64(48.1 \%)$ & $151(61.4 \%)$ \\
\hline Secondary & $16(14.2 \%)$ & $35(26.3 \%)$ & $51(20.7 \%)$ \\
\hline Tertiary & $3(2.7 \%)$ & $17(12.8 \%)$ & $20(8.1 \%)$ \\
\hline None committal & $4(3.5 \%)$ & $2(1.5 \%)$ & $6(2.4 \%)$ \\
\hline
\end{tabular}

\section{Discussion}

Basing on the clinician's report on these cohorts of patients, a number of diseas- 
es were commonly found among the patients such as Typhoid disease caused by Salmonella Typhi which was both experienced by HIV positive and HIV negative patients. However, more of the HIV positive patients had Pneumonia (13.3\%). Diseases experienced by by HIV positive patients were significantly different from those experienced by HIV negative patients $\left(\chi^{2}=27.684, \mathrm{P}=0.024\right)$. There were both mono-infection and multi-infection conditions in both HIV positive and negative patients. However the rates were higher in HIV positive patients than in HIV negative patients apart from the mono-infection of typhoid fever where the rate in HIV positive patients was lower 31 (27.4\%) as compared to the rate in HIV negative patients which was $41(30.8 \%)$. The current study revealed that typhoid fever is an infection that is not only common among the HIV infected patients, but also occur in non HIV infected patients. This is in agreeemt with a study which revealed that the association between HIV and typhoid fever was indistinct [4]. The same study indicated that HIV infection was concomitant with decline in the risk of typhoid fever [4] which is in agreement with the current study that indicates a lower infection of typhoid in HIV positive patients 31 (27.4\%) as compared with HIV negative patients 41 (30.8\%). Study by Meja et al. [19] in Western Kenya, reported that a mong the three common pathogens, the prevalence were; E. coli (22.4\%), Klebsiella pneumonia (10.8\%) and S. aureus (9.1\%). In the current study, Salmonella spp. was $3.1 \%$. A similar study on prevalence study by Saeed et al. [20] reported that Mycobacterium tuberculosis was present in $3.6 \%$ of HIV-infected patients [20].

Conversely, mult-infections recorded a higher rate in HIV positive patients compared to HIV negative patients. This was realized in multiple infection such as Tyhoid and meningitis: in HIV positive patients the rate was $1.8 \%$ as compared to that in HIV negative patients which was $0.0 \%$.

Prevalence of Salmonella spp. among the HIV positive patients in this study was found to be $3.7 \%$. This was slightly higher compared to result obtained by [22], who reported a prevalence of $1.2 \%$ in HIV positive patients in Tanzania. Research by Crump, [22] noted that, HIV is protective against invasive S. typhi infection, hence the low prevalence.

In this study, Klebsiella $s s p$ had an overall prevalence of $18.5 \%$ with a prevalence of $7.4 \%$ in HIV positive and $11.1 \%$ in HIV negative. This was similar to the result obtained by Jung et al. [3] who reported a mortality rate of about $20 \%$ in tertiary care hospitals [3]. However, previous study by Marbou and Kuete [2] in Cameroon established that Klebsiella $s p$. were predominant among HIV patients (29.4\%).

Escherichia coli was not isolated in any of the blood samples analyzed, hence the findings of this current study indicated prevalence of $0.0 \%$ for Escherichia coli.

Brucella spp had a prevalence of $3.70 \%$ in the current research. The low level concurs with the findings from a research by Moreno et al. [17] on HIV patients in Spanish Hospital that showed HIV infection does not increase the incidence 
of Brucellosis.

The present study revealed that the prevalence of enteric bacteria is higher in HIV-infected persons. The anomalies observed in HIV positive patients were probably caused by immunodeficiency due to HIV infection. However, this work also indicated the need for adequate biological follow-up for the best monitoring of infected persons visiting Alupe hospital.

\section{Study Limitations}

Despite the success of the study, there were some limitations that were encountered as follows:

1) Some patients despite meeting the inclusion criteria, declined to take part in the study.

2) The rate of inflow of patients exhibiting health conditions of interest was low hence prolonging the data collection period.

3) The blood culture failing to show growth as much as the antigen test indicated positive results. This was attributed to the fact that, most likely patients might have been on treatmet using antibiotics. These antibiotics might have suppressed bacterial growth in the blood culture. Future studies of this nature should employ BD BACTEC ${ }^{\mathrm{TM}}$ FX Blood Culture System to ensure a maximum growth of bacteria in the blood culture.

4) Some patients were not willing to have their blood be tested for HIV.

\section{Conflicts of Interest}

The authors declare no conflicts of interest regarding the publication of this paper.

\section{References}

[1] Allan, C. and Levine, M.M. (2017) Patterns of Bacteraemia Aetiology. The Lancet Infectious Diseases, 17, 1005-1006. https://doi.org/10.1016/S1473-3099(17)30491-7

[2] Marbou, W.J. and Kuete, V. (2017) Bacterial Resistance and Immunological Profiles in HIV-Infected and Non-Infected Patients at Mbouda Ad Lucem Hospital in Cameroon. Journal of Infection and Public Health, 10, 269-276. https://doi.org/10.1016/j.jiph.2016.04.009

[3] Jung, Y., Lee, M.J., Sin, H.Y., Kim, N.H., Hwang, J.H., Park, J., Choe, P.G., Park, W.B., Kim, E.S., Park, S.W., Park, K.U., Kim, H.B., Kim, N.J., Kim, E.C., Song, K.H. and Oh, M.D. (2012) Differences in Characteristics between Healthcare-Associated and Community-Acquired Infection in Community-Onset Klebsiella Pneumoniae Bloodstream Infection in Korea. BMC Infectious Diseases, 12, Article No. 239. https://doi.org/10.1186/1471-2334-12-239

[4] Keddy, K.H., Sooka, A., Smith, A.M., Musekiwa, A., Tau, N.P., Klugman, K.P., Angulo, F.J. and GERMS-SA (2016) Typhoid Fever in South Africa in an Endemic HIV Setting. PLoS ONE, 11, e0164939. https://doi.org/10.1371/journal.pone.0164939

[5] Janeway Jr, C.A., Travers, P., Walport, M., et al. (2001) Immunobiology: The Immune System in Health and Disease. 5th edition. New York: Garland Science. The Front Line of Host Defense. 
https://www.ncbi.nlm.nih.gov/books/NBK27105/

[6] Reddy, E.A., Shaw, A.V., Crump, J.A. (2010) Community-Acquired Bloodstream Infections in Africa: A Systematic Review and Meta-Analysis. The Lancet Infectious Diseases, 10, 417-432. https://doi.org/10.1016/S1473-3099(10)70072-4

[7] Chuwa, A. (2017) Annual report 2016-2017, Kenya Ministry of Agriculture, Livestock and Fisheries.

[8] Okoye, A.A. and Picker, L.J. (2013) $\mathrm{CD}^{+}$T-cell Depletion in HIV Infection: Mechanisms of Immunological Failure. Immunological Reviews, 254, 54-64. https://doi.org/10.1111/imr.12066

[9] Afessa, B., Morales, I. and Weaver, B. (2001) Bacteremia in Hospitalized Patients with Human Immunodeficiency Virus: A Prospective, Cohort Study. BMC Infectious Diseases, 1, Article No. 13. https://doi.org/10.1186/1471-2334-1-13

[10] Lieberman, J.M. (2003) Appropriate Antibiotic Use and Why It Is Important: The Challenges of Bacterial Resistance. The Pediatric Infectious Disease Journal, 22, 1143-1151. https://doi.org/10.1097/01.inf.0000101851.57263.63

[11] Paterson, D.L. and Bonomo, R.A. (2005) Extended-Spectrum Beta-Lactamases: A Clinical Update. Clinical Microbiology Reviews, 18, 657-686. https://doi.org/10.1128/CMR.18.4.657-686.2005

[12] World Health Organization (2014) Antimicrobial Resistance: Global Report on Surveillance (2014).

http://www.who.int/drugresistance/documents/surveillancereport/en/

[13] Marwa, K.J., Mushi, M.F., Konje, E., Alele, P.E., Kidola, J. and Mirambo, M.M. (2015) Resistance to Cotrimoxazole and Other Antimicrobials among Isolates from HIV/AIDS and Non-HIV/AIDS Patients at Bugando Medical Centre, Mwanza, Tanzania. AIDS Research and Treatment, 2015, Article ID: 103874. https://doi.org/10.1155/2015/103874

[14] Oluyege, A.O., Ojo-Bola, O. and Olagbemi, A.A. (2015) Prevalence and Antibiotic Resistance Pattern of Blood Culture Isolates from Human Immuno-Deficiency Virus (HIV) Patients on Highly Active Anti-Retroviral Therapy (HAART) in Nigeria. African Journal of Microbiology Research, 9, 909-914. https://doi.org/10.5897/AJMR2014.7319

[15] Laupland, K.B. (2013) Incidence of Bloodstream Infection: A Review of Population-Based Studies. Clinical Microbiology and Infection, 19, 492-500. https://doi.org/10.1111/1469-0691.12144

[16] Lameshow, S., Hosmer, D.W., Klar, J., Lwanga, S.K. and World Health Organization (1990) Adequacy of Sample Size in Health Studies. Wiley, Chichester.

[17] Moreno, S., Ariza, J., Espinosa, F.J., Podzamczer, D., Miro, J.M., Rivero, A. and Herrero, F. (1998) Brucellosis in Patients Infected with the Human Immunodeficiency Virus. European Journal of Clinical Microbiology and Infectious Diseases, 17, 319-326. https://doi.org/10.1007/BF01709454

[18] Marschall, J., Fraser, V.J., Doherty, J. and Warren, D.K. (2009) Between Community and Hospital: Healthcare-Associated Gram-Negative Bacteremia among Hospitalized Patients. Infection Control \& Hospital Epidemiology, 30, 1050-1056. https://doi.org/10.1086/606165

[19] Meja, K.C., Mau, I., Pamela, K., David, C., Wilfred, E., Ann, M. and Mosigisi, S.A. (2015) Antibiotic Sensitivity Patterns amongst Post-Mortem Bacterial Isolates from Hiv-Infected Patients in Western Kenya: A Cross Sectional Descriptive Study.

[20] Saeed, N.K., Farid, E. and Jamsheer, A.E. (2015) Prevalence of Opportunistic Infec- 
tions in HIV-Positive Patients in Bahrain: A Four-Year Review (2009-2013). The Journal of Infection in Developing Countries, 9, 060-069.

https://doi.org/10.3855/jidc.4997

[21] Feldman, C. (2005) Pneumonia Associated with HIV Infection. Current Opinion in Infectious Diseases, 18, 165-170.

https://doi.org/10.1097/01.qco.0000160907.79437.5a

[22] Crump, J.A., Ramadhani, H.O., Morrissey, A.B., Msuya, L.J., Yang, L.Y., Chow, S.C. and Kinabo, G.D. (2011) Invasive Bacterial and Fungal Infections among Hospitalized HIV-Infected and HIV-Uninfected Children and Infants in Northern Tanzania. Tropical Medicine \& International Health, 16, 830-837. https://doi.org/10.1111/j.1365-3156.2011.02774.x

[23] Badakhsh, F.F., Carmichael, L.E. and Douglass, J.A. (1982). Improved Rapid Slide Agglutination Test for Presumptive Diagnosis of Canine Brucellosis. Journal of Clinical Microbiology, 15, 286-289. https://doi.org/10.1128/JCM.15.2.286-289.1982 